affected stroke territories, post revascularization mTICI outcomes as well as the pertinent devices are presented and discussed.

Results In our series of 41 patients with emergent small vessel occlusions of the anterior and posterior circulation, 6 ACA and 3 PCA branches occlusions are described. Age on admission was distributed as $68 \pm 16$ years (mean \pm SD), and approximately $51 \%$ were male. mTICI revascularization was possible in $41 \%$ with at least 50\% recanalization in $93 \%$.

Conclusions Mechanical revascularization for emergent small vessel occlusions is a viable intervention alternative with minimal post procedural risk profiles in patients.

Disclosures K. Carr: None. Z. Thwing: None. R. Shivashankar: None. Y. Serulle: None.

\section{E-114 PREDICTORS OF POOR OUTCOME AFTER SUCCESSFUL MECHANICAL THROMBECTOMY OF ANTERIOR CIRCULATION LARGE VESSEL OCCLUSIONS WITHIN 6 HOURS OF SYMPTOM ONSET}

${ }^{1} \mathrm{M}$ Mohammaden*, ${ }^{1} \mathrm{C}$ Stapleton, ${ }^{1} \mathrm{D}$ Brunozzi, ${ }^{2} \mathrm{G}$ Atwal, ${ }^{1} \mathrm{~A}$ Alaraj. 'Neurosurgery, University of Illinois at Chicago, Chicago, IL; ${ }^{2}$ neurosurgery, university of Illinois at Chicago, Chicago, IL

\subsection{6/neurintsurg-2019-SNIS.189}

Introduction Successful recanalization is a significant predictor of a good clinical outcome after mechanical thrombectomy (MT). However, some patients have a poor clinical outcome even with complete revascularization.

Objective We aimed to study the clinical, imaging, and procedural factors that predict a poor clinical outcome (90-day modified Rankin Scale $\geq 3$ ) in patients with anterior circulation large vessel occlusion (LVO) who undergo successful MT within 6 hours of symptom onset.

Patients and methods We performed a retrospective review of all patients who underwent MT within 6 hours of symptom onset for an anterior circulation LVO at our institution between May 2016 to June 2018. Poor outcome was defined as a modified Rankin Scale equal or more to three at 90 days.

Results A total of 56 patients met criteria for inclusion in this study. A poor outcome occurred in 31 (55.4\%) patients. On univariate analysis, compared to patients with good clinical outcomes, patients with poor outcomes had higher mean NIHSS scores on presentation ( 23.3 vs. $13.8, P<0.001)$, were more likely to have internal carotid artery (ICA) occlusions $(38.7 \%$ vs. $8 \%, P=0.008)$, and had a higher incidence of distal clot migration (DCM) after MT $(48.4 \%$ vs. $8 \%, P=0.028)$. Age, gender, other baseline clinical characteristics, the method of revascularization, and incidence of hemorrhagic transformation did not differ between the two cohorts. Using multivariate regression analyses, baseline NIHSS score, site of occlusion, and the presence of DCM remained significant predictors of a poor clinical outcome at 90 days $(P=0.001$, $P=0.026$, and $P=0.04$, respectively).

Conclusion Baseline NIHSS score, ICA occlusion, and DCM are independent predictors of a poor outcome after MT for anterior circulation LVO performed within 6 hours of symptoms onset.

All values are presented as the number of patients (n) and percentage (\%) unless indicated otherwise. Boldface type indicates statistical significance.
Abstract E-114 Table 1 Baseline characteristics, angiographic findings and clinical outcome

\begin{tabular}{|c|c|c|c|}
\hline & $\begin{array}{l}\text { Good Outcome } \\
\mathrm{N}=25\end{array}$ & $\begin{array}{l}\text { Poor Outcome } \\
\mathrm{N}=31\end{array}$ & $P$ value \\
\hline Age (years) & $65.2 \pm 15.1$ & $68.4 \pm 14$ & 0.40 \\
\hline \multicolumn{4}{|l|}{ Mean \pm SD } \\
\hline Gender & $10(40 \%)$ & $17(54.8 \%)$ & 0.27 \\
\hline Atrial fibrillation (AF) & $12(48 \%)$ & $12(38.7 \%)$ & 0.49 \\
\hline Arterial hypertension & $20(78.8 \%)$ & $23(80 \%)$ & 0.61 \\
\hline Diabetes mellitus & $4(16 \%)$ & $7(22.6 \%)$ & 0.54 \\
\hline Time LKW (min) & $215.8 \pm 75$ & $228.2 \pm 77.9$ & 0.55 \\
\hline \multicolumn{4}{|l|}{ Mean \pm SD } \\
\hline ASPECT score (Mean \pm SD) & $9.4 \pm 0.7$ & $9 \pm 1.1$ & 0.14 \\
\hline Prior rTPA administration & $17(68 \%)$ & $23(74.2 \%)$ & 0.61 \\
\hline Baseline NIHSS score & $13.8 \pm 5.7$ & $21.3 \pm 5.3$ & $<0.001$ \\
\hline \multicolumn{4}{|l|}{ Mean \pm SD } \\
\hline Site of occlusion: & $2(8 \%)$ & $12(38.7 \%)$ & 0.008 \\
\hline ICA & $23(92 \%)$ & $19(61.3 \%)$ & \\
\hline \multicolumn{4}{|l|}{ MCA } \\
\hline Tandem lesion & $1(4 \%)$ & $6(19.4 \%)$ & 0.09 \\
\hline Technique & $17(68 \%)$ & $25(80.6 \%)$ & 0.28 \\
\hline Stentriever & $8(32 \%)$ & $6(19.4 \%)$ & \\
\hline \multicolumn{4}{|l|}{ Aspiration } \\
\hline Number of passes & $1.9 \pm 1.4$ & $2.3 \pm 1.8$ & 0.61 \\
\hline \multicolumn{4}{|l|}{ Mean \pm SD } \\
\hline $\begin{array}{l}\text { Puncture to recanalization } \\
\text { (min) Mean } \pm S D\end{array}$ & $37.1 \pm 23.3$ & $51.1 \pm 36.7$ & 0.16 \\
\hline $\mathrm{TICl}$ score $\geq 2 \mathrm{~b}$ & $24(96 \%)$ & $26(83.9 \%)$ & 0.15 \\
\hline Hemorrhagic transformation & $7(28 \%)$ & $14(45.2 \%)$ & 0.19 \\
\hline $\begin{array}{l}\text { Symptomatic hemorrhagic } \\
\text { transformation }\end{array}$ & $2(8 \%)$ & $6(19.4 \%)$ & 0.23 \\
\hline Distal clot migration & $5(20 \%)$ & $15(48.4 \%)$ & 0.028 \\
\hline NIHSS score after procedure & $3.2 \pm 3.7$ & $17.7 \pm 5.5$ & $<0.001$ \\
\hline Mean \pm SD & & & \\
\hline
\end{tabular}

Abstract E-114 Table 2 multivariate analysis for predictors of poor outcome

\begin{tabular}{llll}
\hline & Odds ratio & 95\% Confidence interval & P value \\
\hline Baseline NIHSS score & 1.3 & $1.11-1.52$ & 0.001 \\
Site of occlusion (ICA) & 8.9 & $1.3-60.9$ & 0.026 \\
Distal clot migration (DCM) & 5.77 & $1.09-30.69$ & 0.04 \\
\hline
\end{tabular}

Disclosures M. Mohammaden: None. C. Stapleton: None. D. Brunozzi: None. G. Atwal: None. A. Alaraj: 1; C; NIH. 2; C; Cerenovus.

\section{E-115 INTRACRANIAL SALVAGE TECHNIQUES IN REFRACTORY THROMBECTOMY: WHO, WHEN AND HOW?}

${ }^{1} \mathrm{R}$ Abdalla*, ${ }^{1} \mathrm{M}$ Darwish, ${ }^{1} \mathrm{~T}$ Shokuhfar, ${ }^{1} \mathrm{~S}$ Azmi, ${ }^{2} \mathrm{D}$ Cantrell, ${ }^{2} \mathrm{M}$ Hurley, ${ }^{2} \mathrm{M}$ Potts, ${ }^{2} \mathrm{~B}$ Jahromi, ${ }^{2} \mathrm{~A}$ Shaibani, ${ }^{3} \mathrm{~S}$ Ansari. ${ }^{1}$ Radiology, Northwestern University - Feinberg school of Medicine, Chicago, IL; ${ }^{2}$ Radiology, Neurological Surgery, Northwestern University Feinberg school of Medicine, Chicago, IL; ${ }^{3}$ Radiology, Neurology, Neurological Surgery, Northwestern University - Feinberg school of Medicine, Chicago, IL

\subsection{6/neurintsurg-2019-SNIS.190}

Purpose Emergent large vessel occlusion due to underlying vessel wall pathology may be refractory to the endovascular 